\title{
Geodesics and Torsion Tensor according to g-lift of Riemannian Connection on Cotangent Bundle
}

\section{Rabia CAKAN AKPINAR ${ }^{1 *}$}

\begin{abstract}
In this study, the geodesics and torsion tensor according to g-lift of Riemannian connection $\nabla$ on cotangent bundle $T^{*} M$ are investigated. Firstly, using the components of $\mathrm{g}$-lift of Riemannian connection $\nabla$ on cotangent bundle $T^{*} M$ the components of torsion tensor according to glift of Riemannian connection $\nabla$ are obtained. So the torsion tensor according to g-lift of Riemannian connection $\nabla$ are determined. Finally, geodesics on cotangent bundle $T^{*} M$ according to g-lift of Riemannian connection $\nabla$ are studied.
\end{abstract}

Keywords: Cotangent Bundle, Connection, Torsion Tensor, Geodesic, g-lift.

\section{Kotanjant Demette Riemann Konneksiyonun g-liftine göre Burulma Tensörü ve Geodezikler}

ÖZET: Bu çalışmada, $T^{*} M$ kotanjant demette Riemann konneksiyonun g-liftine göre burulma tensörü ve geodezikler incelenir. İlk olarak, $T^{*} M$ kotanjant demet üzerindeki Riemann konneksiyonun g-liftinin bileşenleri kullanılarak $\nabla$ Riemann konneksiyonun g-liftine göre burulma tensor bileşenleri elde edilir. Böylece $\nabla$ Riemann konneksiyonun g-liftine göre burulma tensörü belirlenir. Son olarak, $\nabla$ Riemann konneksiyonun g-liftine göre $T^{*} M$ kotanjant demet üzerindeki geodezikler çalış1lır.

Anahtar Kelimeler: Kotanjant Demet, Konneksiyon, Burulma Tensörü, Jeodezik, g-lift.

\footnotetext{
${ }^{1}$ Rabia CAKAN AKPINAR (Orcid ID: 0000-0001-9885-6373), Kafkas Üniversitesi, Fen Edebiyat Fakültesi, Matematik Bölümü, Kars, Türkiye

*Sorumlu Yazar/Corresponding Author: Rabia CAKAN AKPINAR, e-mail: rabiacakan@kafkas.edu.tr
} 


\section{INTRODUCTION}

Let $M$ be a smooth manifold and $T^{*} M$ be its cotangent bundle. The various problems of the cotangent bundle are studied by many authors (Mok, 1977; Druta Romaniuc, 2012; Kurek and Mikulski, 2013; Çayır 2019a; Çayır 2019b;). One of basic differential geometric structures on a smooth manifold is geodesic. A geodesic is a curve representing the shortest path between two points in a surface, or more generally in a manifold. The shortest path between two given points in a smooth manifold can defined by using the equation for the length of a curve and then minimizing this length between the points using the calculus variations. The problems of geodesics are studied on the different type tensor bundle of a manifold by some authors. The geodesics in tensor bundle of type $(1, q)$ have been investigated according to the Levi-Civita connection of diagonal lift of $g$ metric (Cengiz and Salimov, 2002). The geodesics in tensor bundle of type $(p, q), p+q>0$, have been investigated according to complete lifts of affine connections (Mağden and Salimov, 2004).

On a smooth manifold endowed with an affine connection, torsion and curvature compose the two basic invariants of the connection. In differential geometry, the notion of torsion is a manner of characterizing a twist or screw of a moving frame around a curve. Torsion can be described concretely as a tensor, or as a vector valued two form on the manifold. If $\nabla$ is an affine connection on a smooth manifold, then the torsion tensor is defined

$$
T(X, Y)=\nabla_{X} Y-\nabla_{Y} X-[X, Y]
$$

where $X, Y$ are vector field and $[X, Y]$ is the Lie bracket of vector fields. The torsion tensors are studied on cotangent bundle of a manifold by many authors (Yano and Patterson, 1967; Mok, 1977).

\section{MATERIALS AND METHODS}

Let $M$ be $n$-dimensional differentiable manifold. Let $T M$ be the tangent bundle of $M$. The local coordinates on $T M$ are $\left(x^{i}, x^{i}\right)=\left(x^{i}, y^{i}\right)$ where $\left(x^{i}\right)$ are local coordinates on $M$ and $\left(y^{i}\right)$ are vector space coordinates according to the basis $\partial / \partial x^{i}$. Let $T^{*} M$ be the cotangent bundle of $M$. The local coordinates on $T^{*} M$ are $\left(x^{i}, x^{i}\right)=\left(x^{i}, p_{i}\right)$ where $\left(x^{i}\right)$ are local coordinates on $M$ and $\left(p_{i}\right)$ are vector space coordinates according to the basis $d x^{i}$. In this paper manifolds, mappings and connection are assumed to be differentiable of class $C^{\infty}$. The indices $i, j, k, \ldots$ have range in $\{1, \ldots, n\}$ and indices $\bar{i}, \bar{j}, \bar{k}, \ldots$ have a range in $\{n+1, \ldots, 2 n\}$.

Let $g$ be a pseudo Riemannian metric. $g^{\sharp}: T^{*} M \rightarrow T M$ is the musical isomorphism associated with $g$ pseudo Riemannian metric with inverse given by $g^{\mathrm{b}}: T M \rightarrow T^{*} M$. Then the musical isomorphisms $g^{\sharp}$ and $g^{\mathrm{b}}$ are given by

$$
g^{\sharp}: x^{M}=\left(x^{m}, x^{\bar{m}}\right)=\left(x^{m}, p_{m}\right) \rightarrow x^{J}=\left(x^{j}, x^{\bar{j}}\right)=\left(\delta_{m}^{j} x^{m}, y^{j}=g^{j m} p_{m}\right)
$$

and

$$
g^{\mathrm{b}}=x^{J}=\left(x^{j}, x^{\bar{j}}\right)=\left(x^{j}, y^{j}\right) \rightarrow x^{M}=\left(x^{m}, x^{\bar{m}}\right)=\left(\delta_{j}^{m} x^{j}, p_{m}=g_{m j} y^{j}\right)
$$


where $g^{i k} g_{k j}=\delta_{j}^{i}$ is the Kronecker symbol. The Jacobian matrices of $g^{\mathrm{b}}$ and $g^{\sharp}$ are given, respectively, by (Cakan et. al., 2016)

$$
\left(g_{*}^{\mathrm{b}}\right)=A=\left(A_{J}^{M}\right)=\left(\begin{array}{cc}
A_{j}^{m} & A_{\bar{j}}^{m} \\
\overline{\bar{m}} & A_{j}^{\bar{m}}
\end{array}\right)=\left(\frac{\partial x^{M}}{\partial x^{J}}\right)=\left(\begin{array}{cc}
\delta_{j}^{m} & 0 \\
y^{s} \partial_{j} g_{m s} & g_{m j}
\end{array}\right)
$$

and

$$
\left(g_{*}^{\sharp}\right)=A=\left(A_{M}^{J}\right)=\left(\begin{array}{cc}
A_{m}^{j} & A_{m}^{j} \\
A_{m}^{\bar{j}} & A_{m}^{\bar{j}}
\end{array}\right)=\left(\frac{\partial x^{J}}{\partial x^{M}}\right)=\left(\begin{array}{cc}
\delta_{m}^{j} & 0 \\
p_{s} \partial_{m} g^{j s} & g^{j m}
\end{array}\right) .
$$

The complete lift on cotangent bundle has been defined and applied to connection in manifold (Yano and Patterson, 1967). The $g$ - lifts of some tensor fields on the cotangent bundle have been defined via musical isomorphism and the $g$ - lifts have been applied to problems of some tensor fields (Salimov and Cakan, 2017). The $g$ - lifts of affine connection and curvature tensor on cotangent bundle have been studied (Cakan and Kemer, 2019). In this paper, we investigate the geodesics and torsion tensor according to the $g$ - lift of the Riemannian connection on cotangent bundle $T^{*} M$.

\section{RESULTS AND DISCUSSION}

Let $M$ be an differentiable manifold and $\nabla$ be symmetric affine connection on $M$. Let ${ }^{C} \nabla^{*}$ be complete lift of symmetric affine connection on cotangent bundle $T^{*} M$. The non-zero components ${ }^{C} \stackrel{\sim}{\Gamma}_{I J}^{K}$ of ${ }^{C} \stackrel{*}{\nabla}$ is given

$$
\begin{aligned}
& { }^{C} \Gamma_{i j}^{*}{ }_{i j}^{k}=\Gamma_{i j}^{k}, \quad C^{C} \Gamma_{i j}^{*} \bar{k}=-\Gamma_{i k}^{j}, \quad{ }^{C} \Gamma_{i j}^{*} \bar{k}=-\Gamma_{k j}^{i} \\
& { }^{C} \Gamma_{i j}^{*} \bar{k}=p_{s}\left(\partial_{k} \Gamma_{i j}^{s}-\partial_{i} \Gamma_{j k}^{s}-\partial_{j} \Gamma_{i k}^{s}+2 \Gamma_{k t}^{s} \Gamma_{i j}^{t}\right)
\end{aligned}
$$

according to where $I, J, \ldots=1, \ldots, 2 n$ (Yano and Ishihara, 1973).

Theorem 1 Let $M$ be a $n$-dimensional pseudo Riemannian manifold with pseudo Riemannian metric $g$. Let ${ }^{C} \nabla$ and ${ }^{C} \nabla$ be complete lifts of $\nabla$ affine connection to $T M$ and $T^{*} M$, respectively. Then the differential of ${ }^{C} \nabla$ by $g^{\text {b }}$, i.e. a $g-\operatorname{lift}^{G}{ }^{*} \nabla$ in the cotangent bundle $T^{*} M$, coincides with the complete lift ${ }^{C} \stackrel{*}{\nabla}$ in the cotangent bundle $T^{*} M$ if $\nabla$ is a Riemannian connection which is a metric connection with vanishing torsion. And the $g-\operatorname{lift}^{G} \nabla^{*}$ has components (Cakan and Kemer, 2019)

$$
\begin{aligned}
& { }^{G} \Gamma_{a b}^{*}=\Gamma_{a b}^{c}, \quad{ }^{G} \Gamma_{a \bar{b}}^{c}=0, \quad{ }^{G} \Gamma_{\bar{a} b}^{c}=0, \quad{ }^{G} \Gamma_{\bar{a} \bar{b}}^{c}=0 \\
& { }^{G} \Gamma_{a b}^{*}{ }_{a b}^{c}=p_{t}\left(\partial_{c} \Gamma_{a b}^{t}-\partial_{a} \Gamma_{b c}^{t}-\partial_{b} \Gamma_{a c}^{t}+2 \Gamma_{c r}^{t} \Gamma_{a b}^{r}\right) \\
& { }^{G} \Gamma_{a \bar{b}}^{*}=-\Gamma_{a c}^{b}, \quad{ }^{G} \Gamma_{\bar{a} b}^{*}=-\Gamma_{c b}^{a}, \quad{ }^{G} \Gamma_{\bar{a} \bar{b}}^{*}=0 .
\end{aligned}
$$

Let $\nabla$ be a Riemannian connection and $T$ be torsion tensor of Riemannian connection $\nabla$ on $M$. Let $\bar{T}$ be torsion tensor of the $g-\operatorname{lift}^{G} \stackrel{*}{\nabla}$ on cotangent bundle $T^{*} M$. Using the components ${ }^{G} \Gamma_{A B}^{*}$ of ${ }^{G} \nabla$ in (1.6) the components $\bar{T}_{A B}^{C}$ of $\bar{T}$ are obtained with the equation

$$
\bar{T}_{A B}^{C}={ }^{G} \Gamma_{A B}^{C}-{ }^{G} \Gamma_{B A}^{C}
$$


according to the induced coordinates $\left(x^{h}, p_{h}\right)$. So we obtain

$$
\begin{aligned}
& \bar{T}_{a b}^{c}={ }^{G} \Gamma_{a b}^{*}{ }^{c}{ }^{G} \Gamma_{b a}^{c}=\Gamma_{a b}^{c}-\Gamma_{b a}^{c}=\Gamma_{a b}^{c}-\Gamma_{a b}^{c}=0 \\
& \bar{T}_{a b}^{c}={ }^{G} \Gamma_{\bar{a} b}^{*}-{ }^{G} \Gamma_{b \bar{a}}^{*}=0 \\
& \bar{T}_{a \bar{b}}^{c}={ }^{G} \Gamma_{a \bar{b}}^{*}{ }^{c}{ }^{G} \Gamma_{\bar{b} a}^{c}=0 \\
& \bar{T}_{\bar{a} b}^{\bar{c}}={ }^{G} \Gamma_{\bar{a} b}^{*}{ }^{\bar{c}}{ }^{G} \Gamma_{b \bar{a}}^{\bar{c}}=-\Gamma_{c b}^{a}-\left(-\Gamma_{b c}^{a}\right)=-\Gamma_{c b}^{a}+\Gamma_{b c}^{a}=-\Gamma_{b c}^{a}+\Gamma_{b c}^{a}=0 \\
& \bar{T}_{a \bar{b}}^{\bar{c}}={ }^{G} \Gamma_{a \bar{b}}^{*}{ }^{\bar{c}}-{ }^{G} \Gamma_{\bar{b} a}^{\bar{c}}=-\Gamma_{a c}^{b}-\left(-\Gamma_{c a}^{b}\right)=-\Gamma_{a c}^{b}+\Gamma_{c a}^{b}=-\Gamma_{c a}^{b}+\Gamma_{c a}^{b}=0 \\
& \bar{T}^{c} \bar{a} \bar{b}={ }^{G} \Gamma^{*}{ }_{\bar{a} \bar{b}}^{c}-{ }^{G} \Gamma^{*} \bar{b} \bar{a}=0 \\
& \bar{T}_{\bar{a} \bar{b}}^{\bar{c}}={ }^{G} \Gamma_{\bar{a} \bar{b}}^{*}{ }^{\bar{c}}{ }^{G} \Gamma_{\bar{b} \bar{a}}^{*} \bar{c}=0 \\
& \bar{T}_{a b}^{\bar{c}}={ }^{G} \Gamma_{a b}^{*}{ }_{a b}{ }^{G} \Gamma_{b a}^{*}{ }_{b a} \\
& =p_{s}\left(\partial_{c} \Gamma_{a b}^{s}-\partial_{a} \Gamma_{b c}^{s}-\partial_{b} \Gamma_{a c}^{s}+2 \Gamma_{c t}^{s} \Gamma_{a b}^{t}\right)-p_{s}\left(\partial_{c} \Gamma_{b a}^{s}-\partial_{b} \Gamma_{a c}^{s}-\partial_{a} \Gamma_{b c}^{s}+2 \Gamma_{c t}^{s} \Gamma_{b a}^{t}\right) \\
& =p_{s} \partial_{c} \Gamma_{a b}^{s}-p_{s} \partial_{a} \Gamma_{b c}^{s}-p_{s} \partial_{b} \Gamma_{a c}^{s}+p_{s} 2 \Gamma_{c t}^{s} \Gamma_{a b}^{t}-p_{s} \partial_{c} \Gamma_{b a}^{s}+p_{s} \partial_{b} \Gamma_{a c}^{s}+p_{s} \partial_{a} \Gamma_{b c}^{s}-p_{s} 2 \Gamma_{c t}^{s} \Gamma_{b a}^{t} \\
& =p_{s} \partial_{c} \Gamma_{a b}^{s}-p_{s} \partial_{c} \Gamma_{b a}^{s}+p_{s} 2 \Gamma_{c t}^{s} \Gamma_{a b}^{t}-p_{s} 2 \Gamma_{c t}^{s} \Gamma_{b a}^{t} \\
& =p_{s} \partial_{c} \Gamma_{a b}^{s}-p_{s} \partial_{c} \Gamma_{a b}^{s}+2 p_{s} \Gamma_{c t}^{s} \Gamma_{a b}^{t}-2 p_{s} \Gamma_{c t}^{s} \Gamma_{a b}^{t}=0 \text {. }
\end{aligned}
$$

Corollary 1 The torsion tensor according to $g-\operatorname{lift}^{G} \nabla^{*}$ of Riemannian connection $\nabla$ is equal to zero. Let $C:[0,1] \rightarrow T^{*} M$ be a curve on cotangent bundle $T^{*} M$. And we suppose that $C$ is expressed locally by $x^{C}=x^{C}(t)$, i.e., $x^{c}=x^{c}(t), x^{\bar{c}}=x^{\bar{c}}(t)=p_{c}(t)$ according to induced coordinates $\left(x^{i}, p_{i}\right)$ on cotangent bundle $T^{*} M . t$ is a parameter.

A curve $C$ on cotangent bundle $T^{*} M$ is a geodesic according to $g-\operatorname{lift}^{G}{ }^{*}$ of a Riemannian connection $\nabla$, when it satisfies the differential equation

$$
\frac{d^{2} x^{C}}{d t^{2}}+{ }^{G} \Gamma_{A B}^{*} \frac{d x^{A}}{d t} \frac{d x^{B}}{d t}=0
$$

according to the induced coordinates $\left(x^{c}, x^{\bar{c}}\right)=\left(x^{c}, p_{c}\right)$.

Using the components of $g-\operatorname{lift}^{G} \stackrel{*}{\nabla}$ we obtain following equations from (1.8):

$$
\begin{gathered}
\frac{d^{2} x^{c}}{d t^{2}}+{ }^{G} \Gamma_{a b}^{*} \frac{d x^{a}}{d t} \frac{d x^{b}}{d t}+{ }^{G} \Gamma_{\frac{a}{a} b}^{*} \frac{d x^{\bar{a}}}{d t} \frac{d x^{b}}{d t}+{ }^{G} \Gamma^{*}{ }_{a \bar{b}}{ }^{c} \frac{d x^{a}}{d t} \frac{d x^{\bar{b}}}{d t}+{ }^{G}{ }^{*} \Gamma_{\bar{a} \bar{b}}^{{ }^{c}} \frac{d x^{\bar{a}}}{d t} \frac{d x^{\bar{b}}}{d t}=0 \\
\frac{d^{2} x^{c}}{d t^{2}}+\Gamma_{a b}^{c} \frac{d x^{a}}{d t} \frac{d x^{b}}{d t}=0
\end{gathered}
$$

and 


$$
\begin{aligned}
& \frac{d^{2} x^{\bar{c}}}{d t^{2}}+{ }^{G} \Gamma^{*}{ }_{a b}^{\bar{c}} \frac{d x^{a}}{d t} \frac{d x^{b}}{d t}+{ }^{G} \Gamma^{*} \frac{\bar{c}}{a b} \frac{d x^{\bar{a}}}{d t} \frac{d x^{b}}{d t}+{ }^{G} \Gamma^{*}{ }_{a \bar{b}}^{\bar{c}} \frac{d x^{a}}{d t} \frac{d x^{\bar{b}}}{d t}+{ }^{G} \Gamma^{*}{ }_{\bar{a}}^{\bar{c}} \frac{d x^{\bar{a}}}{d t} \frac{d x^{\bar{b}}}{d t}=0 \\
& \frac{d^{2} p_{c}}{d t^{2}}+p_{s}\left(\partial_{c} \Gamma_{a b}^{s}-\partial_{a} \Gamma_{b c}^{s}-\partial_{b} \Gamma_{a c}^{s}+2 \Gamma_{c t}^{s} \Gamma_{a b}^{t}\right) \frac{d x^{a}}{d t} \frac{d x^{b}}{d t}-\Gamma_{c b}^{a} \frac{d p_{a}}{d t} \frac{d x^{b}}{d t} \\
& -\Gamma_{a c}^{b} \frac{d x^{a}}{d t} \frac{d p_{b}}{d t}=0 \\
& \frac{d^{2} p_{c}}{d t^{2}}+p_{s} \partial_{c} \Gamma_{a b}^{s} \frac{d x^{a}}{d t} \frac{d x^{b}}{d t}-p_{s} \partial_{a} \Gamma_{b c}^{s} \frac{d x^{a}}{d t} \frac{d x^{b}}{d t}-p_{s} \partial_{b} \Gamma_{a c}^{s} \frac{d x^{a}}{d t} \frac{d x^{b}}{d t} \\
& +2 \Gamma_{c t}^{s} \Gamma_{a b}^{t} \frac{d x^{a}}{d t} \frac{d x^{b}}{d t}-\Gamma_{c b}^{a} \frac{d p_{a}}{d t} \frac{d x^{b}}{d t}-\Gamma_{a c}^{b} \frac{d x^{a}}{d t} \frac{d p_{b}}{d t}=0 \\
& \frac{d^{2} p_{c}}{d t^{2}}-\frac{d}{d t}\left(\Gamma_{a c}^{s} p_{s} \frac{d x^{a}}{d t}\right)-\Gamma_{a c}^{s} \frac{d p_{s}}{d t} \frac{d x^{a}}{d t}+\Gamma_{a c}^{s} \Gamma_{b s}^{m} p_{m} \frac{d x^{a}}{d t} \frac{d x^{b}}{d t} \\
& +p_{s} \partial_{c} \Gamma_{a b}^{s} \frac{d x^{a}}{d t} \frac{d x^{b}}{d t}-p_{s} \partial_{a} \Gamma_{c b}^{s} \frac{d x^{a}}{d t} \frac{d x^{b}}{d t}+p_{s} \Gamma_{c m}^{s} \Gamma_{a b}^{m} \frac{d x^{a}}{d t} \frac{d x^{b}}{d t} \\
& -p_{s} \Gamma_{a m}^{s} \Gamma_{c b}^{m} \frac{d x^{a}}{d t} \frac{d x^{b}}{d t}=0 \\
& \frac{d}{d t}\left(\frac{d p_{c}}{d t}-\Gamma_{a c}^{s} p_{s} \frac{d x^{a}}{d t}\right)-\Gamma_{a c}^{s}\left(\frac{d p_{s}}{d t}-\Gamma_{b s}^{m} p_{m} \frac{d x^{b}}{d t}\right) \frac{d x^{a}}{d t} \\
& +p_{s} R_{c a b}^{s} \frac{d x^{a}}{d t} \frac{d x^{b}}{d t}=0 \\
& \frac{d}{d t}\left(\frac{\delta p_{c}}{d t}\right)-\Gamma_{a c}^{s} \frac{\delta p_{s}}{d t} \frac{d x^{a}}{d t}+p_{s} R_{c a b}^{s} \frac{d x^{a}}{d t} \frac{d x^{b}}{d t}=0 \\
& \frac{\delta^{2} p_{c}}{d t^{2}}+p_{s} R_{c a b}^{s} \frac{d x^{a}}{d t} \frac{d x^{b}}{d t}=0
\end{aligned}
$$

where $\delta p_{c}=d p_{c}-\Gamma_{a c}^{s} p_{s} d x^{a}$. After expressions (1.9) and (1.10) we have

Theorem 2 Let $C$ be a geodesic on cotangent bundle $T^{*} M$ according to the $g-\operatorname{lift}^{G}{ }^{*}$ of Riemannian connection $\nabla$ on $M$. The geodesic $C$ has equations

$$
\begin{aligned}
& \frac{d^{2} x^{c}}{d t^{2}}+\Gamma_{a b}^{c} \frac{d x^{a}}{d t} \frac{d x^{b}}{d t}=0, \\
& \frac{\delta^{2} p_{c}}{d t^{2}}+p_{s} R_{c a b}^{s} \frac{d x^{a}}{d t} \frac{d x^{b}}{d t}=0
\end{aligned}
$$

according to the induced coordinates $\left(x^{c}, p_{c}\right)$ on cotangent bundle $T^{*} M$.

\section{CONCLUSION}

In this paper, The torsion tensor and geodesic are studied according to $g$-lift of Riemannian connection to the cotangent bundle $T^{*} M$. The torsion tensor components and geodesic equations are 
obtained by using components of $g$-lift of Riemannian connection $\nabla$. So the torsion tensor and geodesic according to $g$ - lift of Riemannian connection $\nabla$ are determined on cotangent bundle $T^{*} M$

\section{REFERENCES}

Cakan R, Akbulut K, Salimov A, 2016. Musical Isomorphisms and Problems of Lifts. Chinese Annals of Mathematics Series B, 37(3): 323-330.

Cakan R, Kemer E, 2019. The g-lift of Affine Connection in the Cotangent Bundle. Punjab University Journal of Mathematics, 51(11): 53-62.

Cengiz N, Salimov AA, 2002. Geodesics in the Tensor Bundle of Diagonal LIfts. Haccettepe Journal of Mathematics and Statistics, 31: 1-11.

Çayır H, 2019a. On Integrability Conditions, Operators and the Purity Conditions of the Sasakian Metric with respect to lifts of $F_{\lambda}(7,1)$-structure on the cotangent bundle. Turkish Journal of Mathematics, 43(1): 186-198.

Çayır H, 2019b. Derivatives of Sasakian metric ${ }^{s} g$ on Cotangent Bundle. Communications Faculty Sciences University of Ankara Series A1-Mathematics and Statistics, 68(1): 751-761.

Druta Romaniuc SL, 2012. Natural Diagonal Riemannian Almost Product and Para-Hermitian Cotangent Bundles. Czechoslovak Mathematical Journal, 62(4): 937-949.

Kurek J, Mikulski WM, 2013. The Natural Operators Lifting Connections to Tensor Powers of the Cotangent Bundle. Miskolc Mathematical Notes, 14: 517-524.

Mağden A, Salimov AA, 2004. Geodesics for Complete Lifts of Affine Connections in Tensor Bundles. Applied Mathematics and Computation, 151: 863-868.

Salimov AA, Cakan R, 2017. Problems of $g$-lifts. Proceeding of the Institute of Mathematics and Mechanics, 43(1): 161-170.

Mok KP, 1977. Metrics and Connections on the Cotangent Bundles. Kodai Mathematical Seminar Reports, 28: 226-238.

Yano K, Ishihara S, 1973. Tangent and Cotangent Bundles. Marcel Dekker, New York.

Yano K, Patterson EM, 1967. Vertical and Complete Lifts from a manifold to its Cotangent Bundle. Journal of the Mathematical Society of Japan, 19: 91-113. 